\title{
Corneal Factors Associated with the Amount of Visual Field Damage in Eyes with Newly Diagnosed, Untreated, Open-angle Glaucoma
}

\author{
Gema Bolivar (D) C Cristina Sanchez-Barahona · Samira Ketabi • \\ Vassilios Kozobolis · Miguel A. Teus
}

Received: May 31, 2021 / Accepted: July 1, 2021 / Published online: July 19, 2021

(c) The Author(s) 2021

\section{ABSTRACT}

Introduction: The aim of this study was to evaluate the correlation between several ocular parameters (intraocular pressure [IOP], corneal biomechanical properties) and the visual field (VF) mean deviation (VF MD) in eyes with openangle glaucoma (OAG).

Methods: We conducted a cross-sectional, observational study in which we measured the IOP with Goldmann applanation tonometry, the central corneal thickness (CCT), and the corneal parameters obtained from the Ocular Response Analyzer ${ }^{\circledR}\left(\right.$ ORA $\left.{ }^{\circledR}\right)$ and the Corvis ${ }^{\circledR}$ ST non-contact tonometer, in newly diagnosed

\section{G. Bolivar $(\bowtie) \cdot$ M. A. Teus}

Department of Ophthalmology, University Hospital Principe de Asturias, Carretera Alcalá-Meco S/N, Alcalá de Henares, 28805 Madrid, Spain e-mail: gemabolivardemiguel@yahoo.es

G. Bolivar · S. Ketabi · M. A. Teus

Department of Ophthalmology, University of Alcalá, Alcalá de Henares, Spain

C. Sanchez-Barahona Department of Ophthalmology, University Hospital of Getafe, Madrid, Spain

S. Ketabi

Department of Ophthalmology, University Hospital of Mostoles, Madrid, Spain

\section{Kozobolis}

Department of Ophthalmology, Democritus

University of Thrace, Alexandroupolis, Greece and treatment-naïve eyes with OAG, to investigate whether there was any correlation between these ocular parameters and the VF MD.

Results: A total of 51 eyes were analyzed. A statistically significant correlation was found only between the VF MD and corneal hysteresis $(\mathrm{CH})\left(P=0.003, r^{2}=0.16\right)$ and CCT $(P=0.03$, $\left.r^{2}=0.08\right)$.

Conclusions: These results demonstrate that $\mathrm{CH}$ and CCT are associated with the amount of VF damage in treatment-naïve OAG eyes.

Keywords: Corneal parameters; Examination techniques; Open angle glaucoma; Risk factors; Visual field 


\section{Key Summary Points}

Corneal hysteresis and central corneal thickness can be considered risk factors for defects in the visual field in patients with open-angle glaucoma (OAG).

Only patients with newly diagnosed primary OAG were enrolled in the study (treatment-naïve patients), ensuring that only eyes that had been not previously been treated with any antiglaucoma drugs were included; this was important because ocular hypotensive drugs induce mild changes in the central corneal thickness and in corneal properties.

The results demonstrate that It is important to consider corneal hysteresis in the evaluation of patients with OAG in clinical practice.

\section{INTRODUCTION}

Glaucoma is a multifactorial disease and a relevant cause of visual impairment and blindness worldwide. Both vascular and mechanical factors can contribute to the loss of retinal ganglion cells, which are seen clinically as glaucomatous changes in the optic nerve head and the progression of defects in the visual field (VF) [1]. VF testing provides valuable information about functional damage, which is important for diagnosing glaucoma and even more important for follow-up and management. It is well known that patients with more severe VF defects at diagnosis require a greater reduction of their intraocular pressure (IOP) [2].

Numerous risk factors have been associated with glaucoma, such as age, elevated IOP, family history, thinner central corneal thickness (CCT), and pseudoexfoliation, among others [3].

IOP evaluation has been an essential tool in ophthalmology since the sixteenth century, and the cornea plays an important role in this measurement. The CCT was originally considered to be only a tonometry artifact. However, tonometers have recently been developed that are technically more sophisticated, such as the Ocular Response Analyzer ${ }^{\circledR}$ (ORA; Reichert Technologies, Depew, NY, USA) and the Corvis ${ }^{\circledR}$ ST (Oculus, Wetzlar, Germany). The ORA is a non-contact tonometer that measures the IOP (both the corneal compensated IOP [IOPcc] and the Goldmann correlated IOP [IOPg]) and analyzes corneal viscoelastic behavior, such as corneal hysteresis $(\mathrm{CH})$ and the corneal resistant factor (CRF). These parameters have been evaluated in an earlier study to analyze their association with the severity and progression of glaucoma [4]. The Corvis-ST is also a non-contact tonometer equipped with ultra-high-speed Scheimpflug technology; it provides information on corneal biomechanical behavior. The Corvis-ST Scheimpflug imaging system allows more accurate registration of the corneal deformation process compared with the ORA because it evaluates many parameters related with the corneal deformation [5].

$\mathrm{CH}$ has been associated with glaucoma in that a lower $\mathrm{CH}$ has been related with a more advanced VF defect and with an increased risk for progression. This might be related to a biological link between certain characteristics of some structures in the anterior eye, such as corneal thickness or corneal biomechanical properties, and the structure and deformability of the lamina cribrosa and the peripapillar sclera [6].

The association between $\mathrm{CH}$ and VF defect has been described in patients with OAG and patients suspected of having glaucoma [7]. The authors of some studies have suggested that the risk of progression of VF damage is related to the $\mathrm{CH}$ [8], but all of these studies included patients who were already receiving glaucoma treatment. It is well known that lowering the IOP by either drug therapy or surgery influences the main corneal biomechanical parameters [9]. In addition, some ocular hypotensive drugs induce mild changes in the CCT and corneal properties; for example, topical carbonic anhydrase inhibitors result in a slightly increased CCT and, in contrast, topical prostaglandin analogues 
induce a mildly decreased CCT and increased $\mathrm{CH}[10]$.

In the study reported here, we investigated the correlation between the parameters obtained from the ORA and the Corvis ST and the mean deviation (MD) of the VF, as a measure of VF defect, in treatment-naïve eyes with OAG.

\section{METHODS}

This was an observational, cross-sectional study conducted in the Glaucoma Unit of Hospital Universitario "Príncipe de Asturias" of Alcalá de Henares. A total of 51 patients (51 eyes) with newly diagnosed primary OAG (POAG) were included in the study; consequently no eyes had been treated with antiglaucoma drops before entering the study. The study was conducted according to the Declaration of Helsinki, and informed consent was obtained from patients. The Institutional Review Board (Comité de Ética en Investigación Médica) of the "Hospital Universitario Príncipe de Asturias", approved the study protocol.

POAG was defined as the presence of the typical appearance of glaucomatous optic neuropathy with a corresponding VF defect. The VF was analyzed using the Humphrey Visual Field Analyzer (Carl Zeiss Meditec, Oberkochen, Germany). The white-on-white 24-2 with the Swedish Interactive Threshold Algorithm (SITA) strategy was used in all cases. A minimum of two reliable VF tests was required to obtain a valid VF measurement. If the results of either of the first two VF tests were unreliable or questionable, a third qualifying test was performed, and we selected the last qualifying VF data for analysis.

The IOP value was not used as a criterion for glaucoma diagnosis, therefore the IOP was within normal limits in some patients.

The exclusion criteria were a history of a previous ocular surgery; the presence of any corneal pathology that could affect the VF; history of ocular trauma; an untreated IOP pressure of $>35 \mathrm{mmHg}$; and eyes with a severe VF defect defined as 0 decibel $(\mathrm{dB})$ in one of the four central points or a MD of $<-12 \mathrm{~dB}$. When both eyes of the same patient were eligible, the eye with the lower IOP was selected or, alternatively, the eye with the lower MD score if the IOP values were the same in both eyes. Only patients with newly diagnosed POAG were included, so no eye had been treated with any antiglaucoma drugs before entering the study. Patients taking any systemic medication that could have some effect on the IOP, such as steroids and beta blockers, were excluded.

All patients underwent a complete clinical ocular examination, including measurement of the visual acuity and IOP using Goldmann applanation tonometry, measurement of CCT using ultrasonic pachymetry, and determination of the parameters obtained with the ORA $(\mathrm{CH}$ represents the cornea's viscoelastic response to a rapid air jet; CRF describes the overall viscoelastic resistance of the cornea). The IOPg is the IOP analogous to that measured by Goldmann Applanation Tonometry. The IOPcc is an IOP estimate that uses a mathematical correction to minimize its dependance on corneal viscoelastic properties. The main Corvis ST tonometer parameters include: IOPcorvis (a non-contact tonometry measurement that is based on the first corneal applanation); AT1 (time from the initiation of air-puffing until the first applanation); AL1 (length of the flattened cornea at the first applanation); AV1 (speed of corneal apex in achieving the first applanation); AT2 (time from starting until the second applanation); AL2 (length of the flattened cornea at the second applanation); AV2 (speed of corneal apex achieving the second applanation); HCT (time from initiating airpuffing until the highest concavity of cornea is reached); DA (maximum deformation amplitude of the cornea at the highest concavity; PD (distance of the two apices of the cornea at the time of the highest concavity); CCR (radius of curvature of a circle that fits the corneal concavity at the time of the maximum deformation); and CCT Corvis (measurement of the thinnest CCT with optical pachymetry).

Statistical analyses were conducted using the Pearson correlation coefficient and multiple stepwise regression analysis between the VF defects represented by the MD and the other quantitative variables studied, i.e., Goldmann 
applanation tonometry, IOPcc, IOPg, CH, CRF, CCT, and parameters measured by the Corvis ST. Normality of the data distribution was checked using the Kolmogorov-Smirnov test.

\section{RESULTS}

Fifty-one eyes of 51 patients (33 women, 18 men) with newly diagnosed and treatmentnaïve POAG were recruited.

The mean ( \pm standard deviation) patient age was $70.1 \pm 11.7$ (range 32-98) years, mean VF defect measured by the MD was $-3.4 \pm 3.0$ (range -11.9 to -2.2 ) $\mathrm{dB}$, mean CCT was $546.5 \pm 35.1$ (range 480-614) $\mu \mathrm{m}$, and mean Goldmann applanation tonometry IOP was $21.6 \pm 4.4$ (range $14-32$ ) $\mathrm{mmHg}$. Table 1 shows the results of the analysis.

Gender had no significant effect on the MD. The Pearson correlation test showed that the only statistically significant correlations between the MD and the parameters studied were those between the $\mathrm{MD}$ and the $\mathrm{CH}$ $\left(r^{2}=0.16, P=0.003\right)$ and betwen the MD and CCT $\left(r^{2}=0.08, P=0.03\right)$. Multiple stepwise regression analysis was also performed between the MD and all parameters studied. The only statistically significant correlation remaining was between the $\mathrm{MD}$ and the $\mathrm{CH}\left(r^{2}=0.16\right.$, $P=0.003$ ) (Fig. 1). The correlation analysis also showed that there was a significant correlation between the $\mathrm{CH}$ and CCT $\left(r^{2}=0.22\right.$, $P=0.0004)$.

\section{DISCUSSION}

In this study we analyzed the correlation between corneal viscoelastic properties and the VF defect represented by the MD. The results suggest that there is an inverse relationship between the $\mathrm{CH}$ and the VF defect, i.e., lower $\mathrm{CH}$ values are related to deeper VF defects [4]. Other authors have also reported an association between the $\mathrm{CH}$ and VF defect. Congdon et al. [7] studied patients with POAG and suspected glaucoma, analyzing the influence of corneal biomechanics on the MD, the pattern standard deviation, the Glaucoma Hemifield Test, and VF
Table 1 Goldmann intraocular pressure, central corneal thickness, Ocular Response Analyzer ${ }^{\circledR}$, Corvis ${ }^{\circledR}$ ST, and visual field parameters

\begin{tabular}{|c|c|c|}
\hline Parameter & Mean $\pm S D$ & Range \\
\hline GAT IOP $(\mathrm{mmHg})$ & $21.6 \pm 4.4$ & $14-32$ \\
\hline $\operatorname{CCT}(\mu \mathrm{m})$ & $546.5 \pm 35.1$ & $480-614$ \\
\hline IOPg (mmHg) & $23.11 \pm 7.29$ & $15-46.5$ \\
\hline IOPcc $(\mathrm{mmHg})$ & $24.5 \pm 7.29$ & $13.7-46.4$ \\
\hline $\mathrm{CRF}(\mathrm{mmHg})$ & $10.7 \pm 2.07$ & $6.4-16.2$ \\
\hline $\mathrm{CH}(\mathrm{mmHg})$ & $8.9 \pm 2.32$ & $6.4-16.1$ \\
\hline $\mathrm{AT} 1(\mathrm{~ms})$ & $8.01 \pm 0.73$ & $7.1-10.1$ \\
\hline AT2 (ms) & $20.3 \pm 0.71$ & $17.6-21.3$ \\
\hline $\operatorname{AL1}(\mathrm{mm})$ & $1.7 \pm 0.2$ & $1.4-2.2$ \\
\hline $\operatorname{AL} 2(\mathrm{~mm})$ & $1.8 \pm 0.38$ & $1.2-2.6$ \\
\hline $\operatorname{AV} 1(\mathrm{~m} / \mathrm{s})$ & $0.1 \pm 0.04$ & $0.04-0.3$ \\
\hline $\operatorname{AV} 2(\mathrm{~m} / \mathrm{s})$ & $-0.2 \pm 0.07$ & -0.4 to -0.14 \\
\hline IOP Corvis $(\mathrm{mmHg})$ & $19.3 \pm 5.73$ & $13-37$ \\
\hline CCT Corvis $(\mu \mathrm{m})$ & $530 \pm 37.36$ & $465-641$ \\
\hline $\operatorname{HCT}(\mathrm{ms})$ & $16.5 \pm 0.64$ & $15.2-18.2$ \\
\hline $\mathrm{DA}(\mathrm{mm})$ & $0.8 \pm 0.12$ & $0.5-1.0$ \\
\hline $\mathrm{PD}(\mathrm{mm})$ & $3.4 \pm 1.19$ & $1.4-5.4$ \\
\hline $\mathrm{CCR}(\mathrm{mm})$ & $3.4 \pm 1.58$ & $2.0-11.8$ \\
\hline $\mathrm{MD}(\mathrm{dB})$ & $-3.4 \pm 3.07$ & -11.9 to 2.2 \\
\hline PSD (dB) & $4.08 \pm 2.61$ & $1.68-10.65$ \\
\hline
\end{tabular}

SD Standard deviation, GAT IOP Goldmann applanation tonometry intraocular pressure, $C C T$ central corneal thickness, IOPg Goldmann correlated IOP, IOPcc corneal compensated IOP, $C R F$ corneal resistant factor, $C H$ corneal hysteresis, $A T 1$ time of applanation 1, AT2 time of applanation 2, $A L 1$ length of applanation 1, $A L 2$ length of applanation 2, $A V 1$ velocity of applanation $1, A V 2$ velocity of applanation 2, IOP Corvis Corvis ${ }^{\circledR}$ ST intraocular pressure, CCT Corvis Corvis ${ }^{\circledR}$ ST central corneal thickness, $H C T$ highest concavity, $D A$ deformation amplitude, $P D$ peak distance, $C C R$ central curvature radius, $M D$ mean deviation, $P S D$ pattern standard deviation

progression. Based on their results, they reported only a weak association between the $\mathrm{CH}$ 


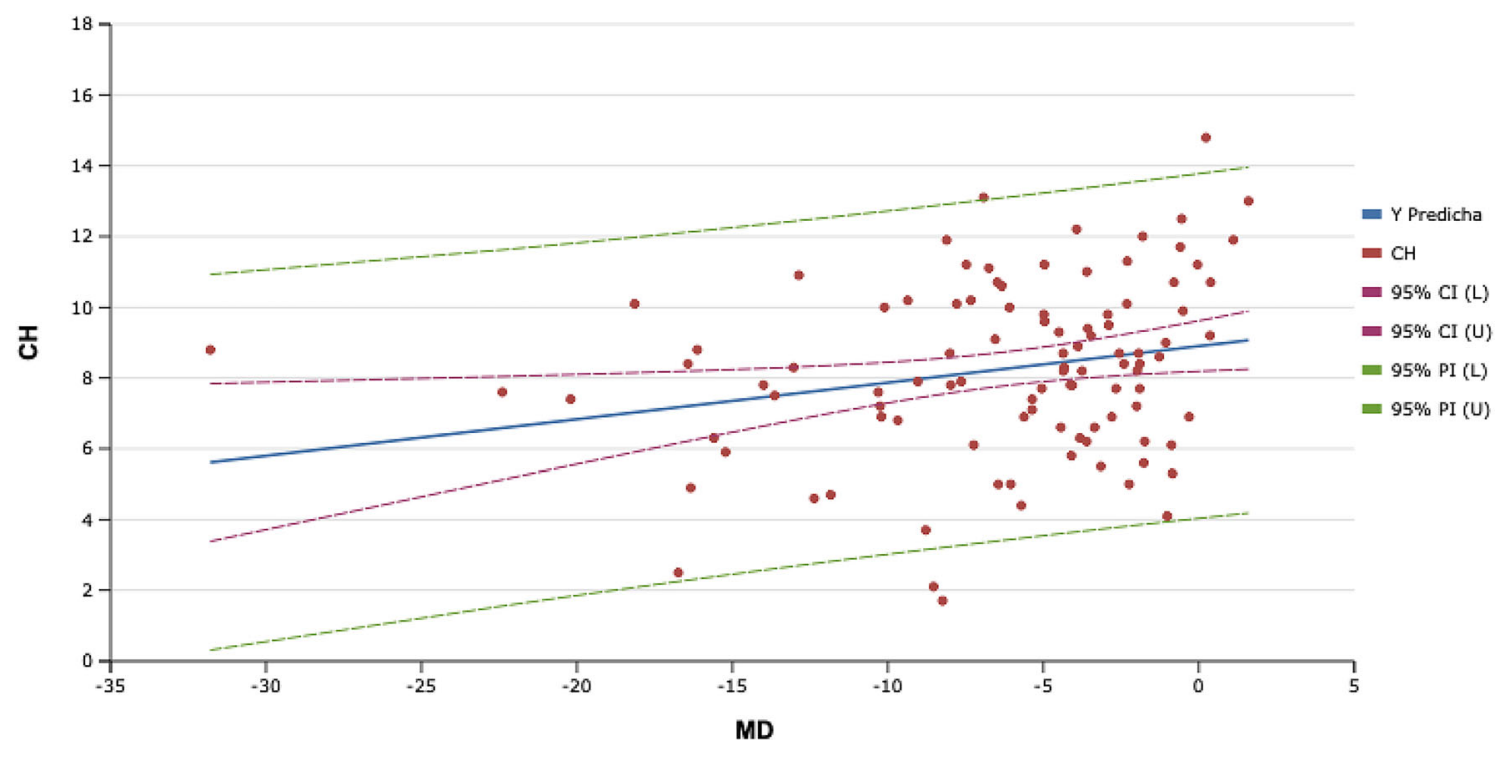

Fig. 1 Graph of the regression analysis between the visual field mean defect (mean deviation $[M D]$ ) and corneal hysteresis $(\mathrm{CH})$

value and VF progression. Anand et al. [11] published a prospective cross-sectional study of patients with asymmetric OAG and reported a statistically significant association between the $\mathrm{CH}$ and VF damage [12]. It is noteworthy that most patients in these studies were receiving chronic IOP-lowering medical therapy prior to the respective study, and it is well known that this kind of treatment can change corneal biomechanical properties $[13,14]$.

The association between corneal parameters and VF defects has also been reported in cohort studies. De Moraes et al. [15] retrospectively evaluated the degree of VF progression and reported a moderate correlation between lower $\mathrm{CH}$ values and thinner CCT. Medeiros et al. [8] reported similar results, but in their case, they prospectively described a formula to predict the amount of change in the VF index based on the $\mathrm{CH}$ values. Nevertheless, two factors may have affected the results of these two studies: (1) disease severity differed between the baseline examination and (2) many patients were already receiving topical treatment prior to the study.

On the other hand, Nongpiur et al. [16] did not find any relationship between corneal viscoelastic properties and VF defects. They also found no association between corneal viscoelastic behavior and glaucoma severity in primary angle-closure glaucoma eyes. However, it is well known that the pathogenesis of primary angle-closure glaucoma differs from that of POAG, which could explain why changes in the eyes over time differed in both groups.

In addition, it is of interest to note that, although the IOP and the $\mathrm{CH}$ are correlatedi.e., the higher the IOP the lower the $\mathrm{CH}$ [9]we found no significant correlation between the IOP level (either Goldmann applanation tonometry, IOPCorvis, or IOPcc) and the MD. In fact, the lack of statistically significant correlation between the untreated IOP and the MD in small cohorts of patients with POAG has been demonstrated years ago [17]. This apparent discrepancy seems to further prove that it is the $\mathrm{CH}$ itself, and not the IOP, which is significantly associated with the amount of VF damage in newly diagnosed patients.

The VF test is an important tool for evaluating glaucomatous damage and is a key factor for diagnosing and following patients with glaucoma. Structural changes in glaucomatous eyes also are relevant, but only a few studies have compared both. $\mathrm{Vu}$ [18] reported that the relation between the $\mathrm{CH}$ and $\mathrm{MD}$ was stronger than 
to structural markers of glaucoma damage as measured by spectral-domain optical coherence tomography. This finding might be explained by the fact that in the early disease the optical coherence tomography structural parameters seem to correlate well with the severity of the disease but that at the later stages it appears that it is the VF damage which shows the stronger correlation with glaucomatous damage.

All previous studies have been performed in patients who were receiving glaucoma treatment prior to entering the study. It is well known that all antiglaucomatous therapies can alter some corneal biomechanical properties, as discussed previously [9]. In the current study, we analyzed only patients with newly diagnosed POAG who had not received any antiglaucoma treatment, so we believe that their biomechanical parameters truly reflected the basal corneal characteristics of each eye.

However, we did not find a significant correlation between the VF parameters and any of the parameters provided by the Corvis ST, possibly because the ORA and the Corvis ST do not measure the same parameters. The ORA provides the $\mathrm{CH}$ value, which reflects the corneal viscoelastic properties, whereas the Corvis ST mainly evaluates corneal stress/strain behavior. In addition, there are differences in the air puff itself between both tonometers [19], which might explain further the correlation between $\mathrm{CH}$ and the VF defect but not between any of the parameters measured by the Corvis ST. Nevertheless, more research is needed in this field. In particular, the newer software provided by the Corvis ST manufacturer, which provides new biomechanical parameters such as the stress-strain index or the biomechanically corrected IOP (bIOP), might be useful, but this software was not available when the current study was performed.

\section{CONCLUSIONS}

The results of this study show that $\mathrm{CH}$ and central CCT are associated with the amount of VF damage in patients newly diagnosed with POAG before the initiation of any treatment. More studies are clearly needed to confirm the importance of corneal biomechanical properties in glaucoma and their possible relationship with VF defects and to evaluate whether the different glaucoma subtypes affect the results. Prospective studies also should evaluate the relation between $\mathrm{CH}$ and $\mathrm{VF}$ progression and the possible effect of glaucoma treatments on these results.

\section{ACKNOWLEDGEMENTS}

We thank the participants of the study.

Funding. No funding or sponsorship was received for this study or publication of this article.

Authorship. All named authors meet the International Committee of Medical Journal Editors (ICMJE) criteria for authorship for this article, take responsibility for the integrity of the work as a whole, and have given their approval for this version to be published.

Authorship Contributions. Gema Bolivar: conception and design of the study, critical revision of the article for important intellectual content, and final approval of the submitted version. Cristina Sanchez-Barahona: acquisition of data, drafting of the article, and final approval of the submitted version. Samira Ketabi: acquisition of data, drafting of the article, and final approval of the submitted version. Vassilios Kozobolis: analysis and interpretation of data, critical revision of the article for important intellectual content, and final approval of the submitted version. Miguel Teus: conception and design of the study, analysis and interpretation of data, critical revision of the article for important intellectual content, and final approval of the submitted version.

Disclosures. Miguel Teus reports receiving grants and personal fees from Johnson \& Johnson, and personal fees and non-financial support from Alcon, Allergan, and Novartis, outside of the submitted work. Gema Bolivar, Cristina Sanchez-Barahona, Samira Ketabi, and Vassilios 
Kozobolis declare that they have no conflict of interest.

Compliance with ethics guidelines. This was an observational, cross-sectional study that was conducted according to the Declaration of Helsinki of 1964. The Institutional Review Board, Comité de Ética en Investigación Médica (CEIm) of the "Hospital Universitario Príncipe de Asturias", approved the study protocol. Informed consent was obtained from all the patients.

Data availability. The datasets generated during and/or analyzed during the current study are available from the corresponding author on reasonable request.

Open Access. This article is licensed under a Creative Commons Attribution-NonCommercial 4.0 International License, which permits any non-commercial use, sharing, adaptation, distribution and reproduction in any medium or format, as long as you give appropriate credit to the original author(s) and the source, provide a link to the Creative Commons licence, and indicate if changes were made. The images or other third party material in this article are included in the article's Creative Commons licence, unless indicated otherwise in a credit line to the material. If material is not included in the article's Creative Commons licence and your intended use is not permitted by statutory regulation or exceeds the permitted use, you will need to obtain permission directly from the copyright holder. To view a copy of this licence, visit http://creativecommons.org/licenses/by$\mathrm{nc} / 4.0 /$.

\section{REFERENCES}

1. Shaarawy TM, Sherwood MB, Hitchings RA, Crowston JG. Shaarawy: glaucoma. In: Volume one: medical diagnosis and therapy. Philadelphia: Saunders Elsevier; 2009.

2. Forchheimer I, de Moraes CG, Teng CC, et al. Baseline mean deviation and rates of visual field change in treated glaucoma patients. Eye (Lond). 2011;25(5):626-32.
3. EGS Foundation. European Glaucoma Society terminology and guidelines for glaucoma, 4th edition-part 1. Br J Ophthalmol. 2017;101(4):1-72.

4. Deol M, Taylor DA, Radcliffe NM. Corneal hysteresis and its relevance to glaucoma. Curr Opin Ophthalmol. 2015;26(2):96-102.

5. Pinero DP, Alcon N. Corneal biomechanics: a review. Clin Exp Optom. 2015;98(2):107-16.

6. Lanzagorta-Aresti A, Perez-Lopez M, Palacios-Pozo E, Davo-Cabrera J. Relationship between corneal hysteresis and lamina cribrosa displacement after medical reduction of intraocular pressure. $\mathrm{Br} \mathrm{J}$ Ophthalmol. 2017;101(3):290.

7. Congdon NG, Broman AT, Bandeen-Roche K, Grover D, Quigley HA. Central corneal thickness and corneal hysteresis associated with glaucoma damage. Am J Ophthalmol. 2006;141(5):868-75.

8. Medeiros FA, Meira-Freitas D, Lisboa R, Kuang TM, Zangwill LM, Weinreb RN. Corneal hysteresis as a risk factor for glaucoma progression: a prospective longitudinal study. Ophthalmology. 2013;120(8): 1533-40.

9. Deol M, Taylor DA, Radcliffe NM. Corneal hysteresiss and its relevance to glaucoma. Curr Opin Ophthalmol. 2015;26(2):96-102.

10. Bolivar G, Sanchez-Barahona C, Teus M, Castejon MA, Paz-Moreno-Arrones J, Gutierrez-Ortiz C, et al. Effect of topical prostaglandin analogues on corneal hysteresis. Acta Ophthalmol. 2015;93(6):e495-8.

11. Anand A, De Moraes CG, Teng CC, Tello C, Liebmann JM, Ritch R. Corneal hysteresis and visual field asymmetry in open angle glaucoma. Invest Ophthalmol Vis Sci. 2010;51(12):6514-8.

12. [No authors listed]. Advanced glaucoma intervention study. 2. Visual field test scoring and reliability. Ophthalmology 1994;1445-55.

13. Inoue $\mathrm{K}$, Okugawa $\mathrm{K}$, Oshika $\mathrm{T}$, Amano S. Influence of dorzolamide on corneal endothelium. Jpn J Ophthalmol. 2003;47(2):129-33.

14. Sawada A, Yamamoto T, Takatsuka N. Randomized crossover study of latanoprost and travoprost in eyes with open-angle glaucoma. Graefes Arch Clin Exp Ophthalmol. 2012;250(1):123-9.

15. De Moraes CV, Hill V, Tello C, Liebmann JM, Ritch $\mathrm{R}$. Lower corneal hysteresis is associated with more rapid glaucomatous visual field progression. J Glaucoma. 2012;21(4):209-13.

16. Nongpiur ME, Png O, Chiew JW, et al. Lack of association between corneal hysteresis and corneal 
resistance factor with glaucoma severity in primary angle closure glaucoma. Invest Ophthalmol Vis Sci. 2015;56(11):6879-85.

17. Teus MA, Castejon MA, Calvo MA, Perez-Salaices P, Marcos A. Intraocular pressure as a risk factor for visual field loss in pseudoexfoliative and primary open-angle glaucoma. Ophthalmology. 1998;105: 2225-30.
18. Vu DM, Silva FQ, Haseltine SJ, Ehrlich JR, Radcliffe NM. Relationship between corneal hysteresis and optic nerve parameters measured with spectral domain optical coherence tomography. Graefes Arch Clin Exp Ophthalmol. 2013;251(7):1777-83.

19. Roberts CJ. Concepts and misconceptions in corneal biomechanics. Corneal Biomech. 2014;40(6): 862-9. 